\title{
Effects of partial versus complete lesions of the amygdala on cross-modal associations in cynomolgus monkeys
}

\author{
LUDIŠE MÁLKOVÁ and ELISABETH A. MURRAY \\ National Institute of Mental Health, Bethesda, Maryland
}

\begin{abstract}
Aspiration lesions of the amygdala plus subjacent cortex were found earlier to produce a severe impairment in cross-modal (tactual-to-visual) recognition (Murray \& Mishkin, 1985). To determine whether more selective lesions would also produce this effect, we trained 8 naive cynomolgus monkeys on a tactual-visual version of delayed nonmatching-to-sample and then injected ibotenic acid bilaterally into either the basolateral $(n=4)$ or centromedial $(n=4)$ subdivisions of the amygdala Neither of the excitotoxic lesions affected performance. In a second experiment, we aspirated the amygdala plus subjacent cortex in similarly trained monkeys. The performance of these animals fell significantly and remained substantially below preoperative levels despite extensive postoperative retraining. The findings suggest that the severe deficit in cross-modal recognition following the aspiration lesion is attributable to complete amygdala damage, damage to the subjacent cortex, or the two in combination.
\end{abstract}

That monkeys can perform cross-modal recognition was conclusively demonstrated first by Cowey and Weiskrantz (1975; Weiskrantz \& Cowey, 1975) and subsequently confirmed by others (Bolster, 1978; Elliot, 1977; Jarvis \& Ettlinger, 1977). The specific neural substrates conferring this ability, however, are still unknown. In early studies that sought to uncover the neural substrate (e.g., Petrides \& Iversen, 1976), the results were ambiguous because the sensory and memory abilities within each modality had not been assessed independently of the cross-modal ability. To address this problem, Jarvis and Ettlinger (1977) devised a test that called not only for cross-modal recognition but also for discrimination of objects within each of the two sensory modalities separately. In their task, monkeys were trained on a series of two-choice object discrimination problems, alternate problems being presented in the visual and tactual modalities. Attainment of criterion on each pair was followed immediately by a single choice trial in the other modality with the same pair of objects. Using this method, Streicher and Ettlinger (1987) found that monkeys with subtotal aspiration lesions of the amygdala had, at most, a transient impairment in cross-modal abilities, a finding that appeared to be at odds with an earlier one by Murray and Mishkin (1985), indicating that complete aspira-

The authors thank Sandra Angeli, Elizabeth Gerpe, Cathy Henderson, and Jeff Martone for their valuable help in testing monkeys, and Jimmy Grant, David Musci, David Kramer, and Ray Phillips for assisting in surgery. In addition, the authors thank Bill Balew for performing the radiographs, and Mortimer Mishkin for his support in all phases of this study. Correspondence should be addressed to E. A. Murray, Laboratory of Neuropsychology, National Institute of Mental Health, Bldg.49, Rm. 1B80, Bethesda, MD 20892 (e-mail: eam@) In.nimh.nih.gov). tion lesions of the amygdala (that included damage to subjacent cortex) produced a severe impairment. In the latter study, monkeys had been trained postoperatively with a set of 40 familiar objects using a cross-modal (tactual-to-visual) version of delayed nonmatching-tosample (DNMS). A baited sample object was presented for familiarization in the dark followed by a choice test, in which the sample object, now unbaited, was presented with an alternative, baited, object in the light. Hippocampectomized monkeys succeeded on the task, whereas amygdalectomized monkeys performed at near-chance levels, even though they performed accurately on both intramodal versions of the task with the same set of objects and the same sample-test delay intervals $(10 \mathrm{sec})$.

In an attempt to resolve the discrepancy in the results of the two studies described above, we first assessed the effects of subtotal lesions of the amygdala, as Streicher and Ettlinger (1987) had done, but used the task employed by Murray and Mishkin (1985). Because the results were negative, we performed a second experiment using the same task, but with monkeys given the large aspiration lesions.

\section{EXPERIMENT 1}

The anatomical connections of the basolateral amygdala appear to make it a prime candidate for linking sensory and mnemonic information across modalities. This subdivision receives the bulk of the amygdalopetal projections arising in modality-specific neocortical areas (Aggleton, Burton, \& Passingham, 1980; Amaral, Insausti, \& Cowan, 1983; Amaral \& Price, 1984; Friedman, Murray, O’Neill, \& Mishkin, 1986; Herzog \& Van Hoesen, 1976; Iwai \& Yukie, 1987; Mufson, Mesulam, \& Pandya, 1981; Turner, Mishkin, \& Knapp, 1980; Van Hoesen, 
1981 ) and is connected with the medial dorsal nucleus of the thalamus (Aggleton \& Mishkin, 1984; Russchen, Amaral, \& Price, 1987), which has been implicated in stimulus memory (Aggleton \& Mishkin, 1983; Gaffan \& Murray, 1990; Zola-Morgan \& Squire, 1985). Furthermore, the lateral basal nucleus, in particular, receives heavy inputs from higher order modality-specific neocortical areas and also gives rise to reciprocal projections to the neocortex (Amaral \& Price, 1984; Barbas \& de Olmos, 1990; Friedman et al., 1986; Iwai \& Yukie, 1987; Morecraft, Geula, \& Mesulam, 1992; Mufson et al., 1981; Tigges et al., 1982); this nucleus could, therefore, be responsible for mediating cross-modal associations. To test this possibility, we assessed the effects of excitotoxic lesions of the basolateral amygdala and compared them with those of excitotoxic damage to the centromedial amygdala, the other major subdivision of the amygdaloid complex.

\section{Method}

\section{Subjects}

The subjects were 8 experimentally naive cynomolgus monkeys (Macaca fascicularis): 1 female and 7 males, weighing $2.8-4.7 \mathrm{~kg}$ at the beginning of the study. They were housed individually in rooms with automatically regulated lighting (12:12 h light:dark) and were maintained on a diet of Purina monkey chow supplemented with fresh fruit. Water was always available.

\section{Apparatus and Materials}

Behavioral testing was carried out in a Wisconsin General Testing Apparatus (WGTA), which was located inside a darkened, sound-shielded room. Additional sound masking was provided by a white-noise generator. The test compartment of the WGTA was fitted with a $60-W$ incandescent bulb that could be dimmed by use of a variable resistor. A one-way vision screen allowed observation of the animal's responses during testing in the light. During testing in the dark, the one-way screen was covered by an opaque screen. The animal's behavior in the dark was observed on a video monitor linked to an infrared camera which, together with an infrared light source, was mounted over the test compartment. The test tray, painted black, contained a row of three food wells spaced $15 \mathrm{~cm}$ apart, center to center. Light-emitting diodes, recessed in the test tray $4.5 \mathrm{~cm}$ in front of each well, were used to signal the position of the objects during tactual testing, but they did not provide sufficient light for either localization or identification of the objects.

The monkeys were trained with a fixed set of 40 visually and tactually distinctive objects that differed widely in shape, size, texture, and compressibility. Each object was mounted on a cork base that fit tightly into the wells of the test tray. This forced the monkeys to grasp and lift and, thus, palpate the objects when displacing them. The same set of objects was used successively in the visual, tactual, and cross-modal stages of the task (see Preoperative Training section, below).

Food rewards consisted of 300-mg banana-flavored pellets (P. J. Noyes Co.).

\section{Preoperative Training}

Pretraining. The monkeys were first trained by successive approximation to displace a gray plaque that covered the one (of the three) wells that had been baited on that trial. The location of the baited well (left, right, center) followed a random order. During pretraining, the monkeys' spontaneous hand preferences were noted, and they were trained to use their preferred hands only, to ensure that all tactual comparisons would be intramanual. After each monkey had learned to use its preferred hand consistently, it began formal training.

Stage 1: Delayed nonmatching-to-sample in the light. During Stage 1, the test compartment was always illuminated. First, a sample object was presented over the central well, which the monkey could displace to obtain a food reward hidden underneath. After a 10-sec delay, the sample was presented again together with a new object, each over a lateral well, for choice. The monkey could obtain another food reward by displacing the new object. After a $30-\sec$ intertrial interval, another trial was given in the same way but with a new pair of objects, and so on, for the 20 trials composing a test session. Each of the 40 objects was used in only 1 trial per daily session, and the objects were randomly paired again before each session. There was no correction after errors. Initially, the cork bases of the objects were inserted in the wells only partly and at an angle, so that the monkeys could displace the stimuli easily. As training progressed, the cork bases were inserted more deeply into the wells, forcing the animal to grasp the objects firmly and thereby obtain tactile information before removing them and procuring the food reward. The monkeys were trained on this initial task, visual DNMS, to the criterion of 90 correct responses in 100 consecutive trials.

Stage 2: Delayed nonmatching-to-sample in the dark. After attaining criterion on visuai DNMS, the monkeys were taken through a light-dimming phase in order to introduce them to tactual DNMS. During this phase, the light in the test compartment was dimmed progressively according to a titration schedule as follows. Light was reduced in 12 steps, with initially large reductions in lamp voltage followed by smaller reductions. If a monkey scored 8 or more correct responses in a block of 10 trials, the light level was reduced 1 step; if the monkey scored 7 or fewer correct out of 10 , the light remained at the same level, though for no more than 2 days, at which time the light was lowered again irrespective of the score. In this way, the light level was gradually reduced from $0.95 \mathrm{fL}$ (the normal light level in the test compartment) to $0.0 \mathrm{fL}$. During the light-dimming phase, a correction procedure was introduced in which each incorrect response was followed by one or more re-presentations of the entire trial sequence (sample presentation plus choice test) until the animal chose correctly. This correction procedure was used throughout the light-dimming and tactual-testing phases. Training on tactual DNMS continued until the animal reached the same criterion as in the light (i.e., 90 correct responses in 100 trials distributed across 5 consecutive days).

Stage 3: Tactual-to-visual delayed nonmatching-to-sample. Once the monkeys had mastered tactual DNMS, they were transferred to the final, cross-modal, condition. In this phase, the sample object was presented in the dark, but the choice test was given in the light. To ensure that the monkeys' choices were made using visual cues only, the first object touched was scored as the response, and the correction procedure was eliminated. Training continued at the rate of 20 trials per daily session, and the same delay and intertrial intervals were used as before. Each animal received a minimum of 400 trials, at which point it was considered to have solved the task if it achieved a mean of $75 \%$ correct responses or better across the 400-trial block. Only 3 monkeys (BL-4, CM-3, and CM-4; see Surgery section) achieved this level of performance. The 5 remaining monkeys therefore were given additional training with one of two remedial methods. Two monkeys (BL-3 and CM-2) received 200 trials with light-dimming training (see Stage 2) followed by retesting on cross-modal DNMS for 400 trials, during which they reached the criterion. The three other monkeys (BL-1, CM-1, and BL-2) received 500 additional trials on cross-modal DNMS with double presentation of the sample. In this procedure, the two presentations of the sample were separated by a 10 -sec interval, and reward was available for the first presentation only. All 3 monkeys achieved criterion in the last 400 trials, after which 
Table 1

Percent Damage to Basolateral (BL) and Centromedial (CM) Amygdala

\begin{tabular}{lrr}
\hline Subject & BL & CM \\
\hline BL-1 & 16 & 2 \\
BL-2 & 17 & 1 \\
BL-3 & 18 & 0 \\
CM-1 & 7 & 63 \\
CM-2 & 5 & 43 \\
CM-3 & 6 & 37 \\
\hline
\end{tabular}

Note-Numerals indicate the mean damage sustained in the two hemispheres, expressed as a percent of the total volume of the nuclear group.

they received 300 trials with single-sample presentation. Finally, to determine whether cross-modal performance was stable over time, 1 monkey that learned the cross-modal task quickly (CM-4) and 1 that required remedial training (BL-2) were each given an additional 400 trials on the cross-modal task before surgery. Scores obtained on the last 300 trials before surgery were used as the preoperative baseline (see Table 3 below).

\section{Postoperative Testing}

Two to 6 weeks after surgery, the monkeys were retested on cross-modal DNMS, in the same manner as for the preoperative baseline, for a total of 500 trials.

\section{Surgery}

After completing preoperative training, the animals were divided into two surgical groups that were balanced for preoperative learning and performance scores. Four monkeys received bilaterally symmetrical injections of ibotenic acid aimed at the basolateral nuclei of the amygdala (Group BL), and the 4 remaining monkeys received injections aimed at the centromedial nuclei of the amygdala (Group CM).

Prior to surgery, each monkey was anesthetized and placed in a stereotaxic frame in order to obtain X-rays of the head. The coordinates for injections were calculated on the basis of the positions of the sphenoid bone and posterior clinoid process, as determined from the X-ray films in the sagittal plane (Aggleton, 1985; Aggleton \& Passingham, 1981). The injection sites were separated by approximately $2 \mathrm{~mm}$ in each plane and were intended to allow diffusion of the excitotoxin ibotenic acid (Sigma Chemical, St. Louis) throughout either the basolateral or the centromedial amygdala.

At surgery, the monkey was anesthetized with an initial dose of ketamine hydrochloride $(10 \mathrm{mg} / \mathrm{kg})$ followed by Nembutal, which was administered intravenously as required (approximately 20 $30 \mathrm{mg} / \mathrm{kg}$ ), and placed in a stereotaxic frame. The animal also received an intravenous drip ( $5 \%$ dextrose and $0.45 \%$ sodium chloride), and its heart rate, respiration rate, and temperature were monitored. Aseptic techniques were used throughout the surgery, which was performed bilaterally in one stage. A bone flap was made in the appropriate portion of the cranium and small slits were cut in the dura to allow the needle of a $10-\mu 1$ Hamilton syringe, held in a Kopf electrode manipulator, to be lowered to the proper coordinates. The 4 monkeys in Group BL received 9 or 10 injections per hemisphere, and the 4 monkeys in Group CM received 4 or 5 injections per hemisphere. A total of $0.4-\mu 1$ ibotenic acid $(10-15 \mathrm{mg} / \mathrm{ml})$ was injected through a 30 -ga needle at each site. In order to allow diffusion of the ibotenic acid into the extracellular space and to minimize mechanical damage to the tissue, all injections were made at a rate of $0.2 \mu \mathrm{l}$ per minute. After the injections were completed, the scalp was closed in anatomical layers, and an antibiotic was administered as a prophylactic measure.

\section{Histology}

On completion of the experiment, 3 of the 4 monkeys in each group were given an overdose of barbiturates (sodium pentobarbital, $100 \mathrm{mg} / \mathrm{kg}$, i.p.) and were perfused through the heart with normal saline followed by aldehyde fixatives. (The 2 remaining monkeys participated in Experiment 2.) The brains were removed, embedded in celloidin, and sectioned at $25 \mu \mathrm{m}$ in the coronal plane. Every 10 th or 20 th section was stained with thionin, mounted, and coverslipped.

The extent of damage to basolateral and centromedial amygdala in each monkey is indicated in Table 1 , and the location and extent of the damage in two representative cases of each group is illustrated in Figure 1. In general, the lesions were smaller than intended. Monkeys in Group CM sustained an average of $48 \%$ damage to the centromedial amygdala and $6 \%$ damage to the basolateral amygdala. Monkeys in Group BL sustained an average of only $17 \%$ damage to the basolateral group of the amygdala and $7 \%$ to the centromedial amygdala. Within the basolateral group, the lateral basal nucleus sustained substantial damage bilaterally $(70 \%)$ in 1 monkey $(\mathrm{BL}-1)$ but to a lesser amount in the other $3($ mean $=27 \%)$.

As for unintended damage, 1 monkey (BL-2) sustained slight cell loss in the hippocampus and rhinal cortex. The hippocampal damage affected mainly the anterior portion of the CA3 fields and subiculum bilaterally. The rhinal cortex damage involved the fundus of the left rhinal sulcus, underlying the anterior hippocampus, approximately $4 \mathrm{~mm}$ in anteroposterior extent. Another monkey (BL-3) had extensive damage to the head of the caudate nucleus and anterior dorsal putamen on the right, presumably due to infarction along the track of the needle. This monkey also had cell loss in the deep layers of the anterior entorhinal cortex that extended roughly $2 \mathrm{~mm}$ on the right side and $3 \mathrm{~mm}$ on the left but amounting to no more than $5 \%$ of the entire volume of the entorhinal cortex.

\section{Results}

\section{Preoperative Learning and Performance}

The monkeys required an average of 720 trials (range $=$ 540-960) and 151 errors (range $=87-231$ ) to complete visual (Stage 1) and tactual (Stage 2) DNMS training (Table 2). As intended, during the light-dimming phase, all monkeys learned that they could touch both objects in succession before choosing, and they continued doing so when no light was available (i.e., during tactual DNMS). The monkeys made tactual comparisons on $53 \%$ (range = $46 \%-59 \%$ ) of the trials during the last 100 trials of training on tactual DNMS, confirming their use of tactile cues to solve the task.

As indicated in the Method section, successful transfer to the cross-modal version of DNMS was achieved by 3 monkeys (BL-4, CM-3, and CM-4) within the first 400 trials, but the other 5 monkeys obtained mean scores below $75 \%$ and so were given additional training. The 8 monkeys received a mean total of 650 trials on crossmodal DNMS, and they scored an average of $80 \%$ correct responses over the last 300 trials (see Table 3). An analysis of variance (ANOVA) indicated that the two surgical groups that were formed did not differ in either the total amount of training they had received or their preoperative performance levels on the cross-modal task.

\section{Postoperative Performance}

Comparison between the preoperative and postoperative scores in a group $\times$ condition (pre-post) ANOVA, 

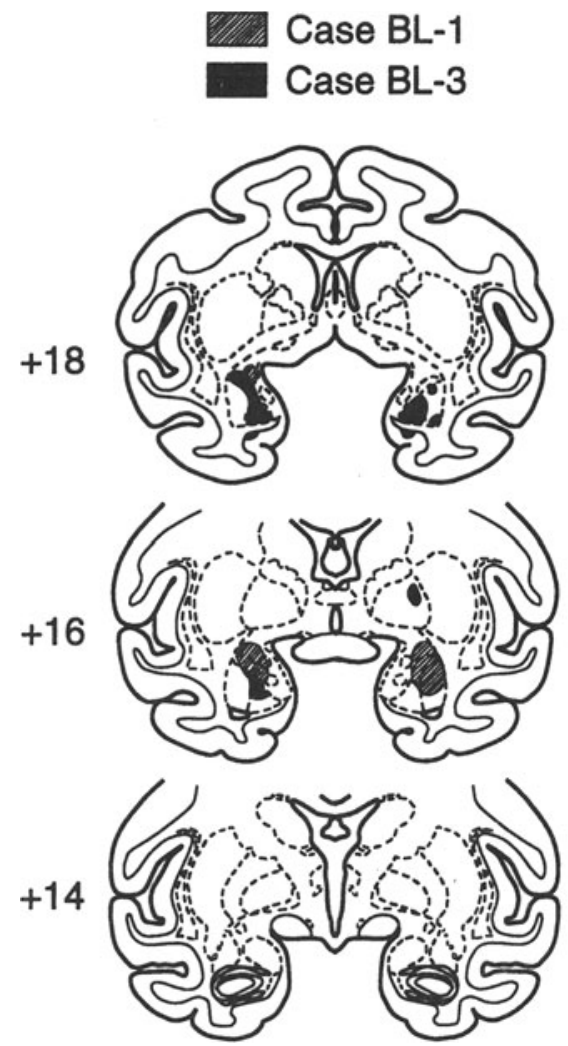
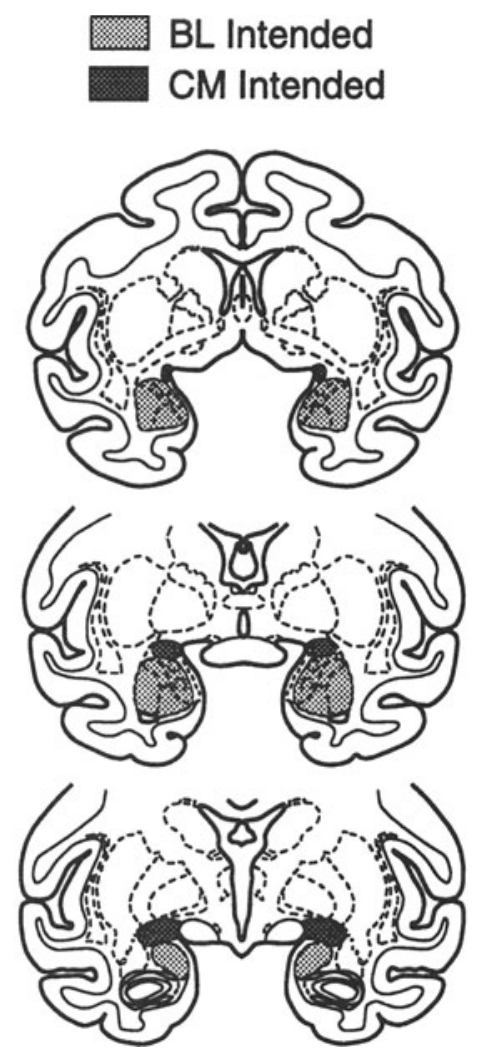
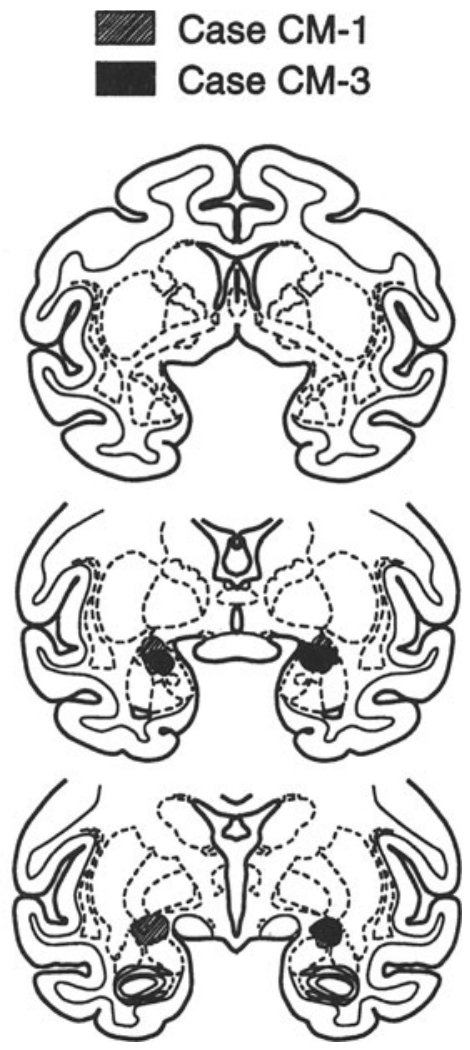

Figure 1. Coronal sections showing the intended lesions (middle column) of the basolateral (BL) and the centromedial (CM) subdivisions of the amygdala, and the extent of damage in two representative cases from each group in Experiment 1 , the basolateral group (BL-1, BL-3; left column) and the centromedial group (CM-1, CM-3; right column). Numerals indicate the distance in millimeters from the interaural plane (0).

with the latter factor as a repeated measure, failed to reveal a significant effect of group, condition, or interaction between the two factors.

\section{Comment}

The results of Experiment 1 are consistent with the findings of Streicher and Ettlinger (1987). In neither study did subtotal lesions of the amygdala yield an impairment in cross-modal (tactual-visual) association. In the study by Murray and Mishkin (1985), by contrast, complete ablation of the amygdala plus subjacent cortex had yielded a severe impairment on the task used in the present investigation. The difference in outcome could have been due to the difference in the locus or extent of the lesions in the three studies. Another possibility, however, is that the difference was due to differences in the training regimen. In the Murray and Mishkin (1985) study, unlike in the present Experiment 1, the monkeys were trained on both visual and tactual DNMS preoperatively, retested first on tactual followed by visual DNMS postoperatively, and only then confronted, for the first time, with the crossmodal version of DNMS. Consequently, the lack of impairment in Experiment 1 could have been due to the use of a less sensitive measure of postoperative impairment-namely, postoperative retention as compared with postoperative acquisition.

\section{EXPERIMENT 2}

To determine whether the negative results in Experiment 1 were due to the ineffectiveness of the subtotal amygdala lesions or to the insensitivity of the preoperative training procedure, we assessed the effect of amyg-

Table 2

Preoperative Learning

\begin{tabular}{|c|c|c|c|c|c|c|}
\hline \multirow[b]{2}{*}{ Lesion } & \multicolumn{2}{|c|}{ Visual DNMS } & \multicolumn{2}{|c|}{ Tactual DNMS } & \multicolumn{2}{|c|}{$\begin{array}{l}\text { Cross-Modal } \\
\text { DNMS }\end{array}$} \\
\hline & Trials & Errors & Trials & Errors & Trials & Errors \\
\hline BL-1 & 340 & 92 & 400 & 90 & 900 & $204^{*}$ \\
\hline BL-2 & 240 & 31 & 400 & 56 & 1,300 & $338^{*}$ \\
\hline BL-3 & 360 & 74 & 580 & 157 & 500 & $188+$ \\
\hline BL-4 & 180 & 37 & 360 & 51 & 100 & 17 \\
\hline$M$ & 280 & 59 & 435 & 89 & 750 & 187 \\
\hline $\mathrm{CM}-1$ & 200 & 49 & 520 & 119 & 900 & $328^{*}$ \\
\hline $\mathrm{CM}-2$ & 280 & 66 & 320 & 61 & 500 & $131+$ \\
\hline $\mathrm{CM}-3$ & 480 & 98 & 480 & 87 & 100 & 22 \\
\hline $\mathrm{CM}-4$ & 220 & 58 & 400 & 80 & 500 & 88 \\
\hline$M$ & 295 & 68 & 430 & 87 & 550 & 142 \\
\hline
\end{tabular}

Note-The total number of trials and errors scored in the three stages of training, excluding the 300-trial preoperative baseline. *Includes 500 trials with double-sample presentation, †Received 200 additional trials with light-dimming phase before attaining criterion on cross-modal DNMS (not included in table). 
Table 3

Performance on Cross-Modal DNMS

\begin{tabular}{|c|c|c|c|c|c|c|c|c|c|c|}
\hline \multirow[b]{2}{*}{ Lesion } & \multicolumn{4}{|c|}{$\begin{array}{l}\text { Preoperative Performance } \\
\text { in Last } 300 \text { Trials } \\
\end{array}$} & \multicolumn{6}{|c|}{ Postoperative Performance } \\
\hline & 100 & 200 & 300 & $M$ & 100 & 200 & 300 & 400 & 500 & $M$ \\
\hline BL-1 & 81 & 92 & 85 & 86 & 81 & 82 & 84 & 86 & 91 & 85 \\
\hline BL-2 & 66 & 76 & 71 & 71 & 70 & 73 & 71 & 82 & 80 & 75 \\
\hline BL-3 & 75 & 80 & 78 & 78 & 74 & 61 & 68 & 62 & 67 & 66 \\
\hline BL-4 & 89 & 83 & 88 & 87 & 88 & 93 & 82 & 92 & 89 & 89 \\
\hline$M$ & 78 & 83 & 81 & 81 & 78 & 77 & 76 & 81 & 82 & 79 \\
\hline $\mathrm{CM}-1$ & 72 & 76 & 76 & 75 & 70 & 68 & 76 & 71 & 72 & 71 \\
\hline $\mathrm{CM}-2$ & 79 & 82 & 81 & 81 & 82 & 85 & 86 & 81 & 84 & 84 \\
\hline $\mathrm{CM}-3$ & 75 & 74 & 79 & 76 & 69 & 72 & 82 & 79 & 82 & 77 \\
\hline $\mathrm{CM}-4$ & 86 & 81 & 85 & 84 & 81 & 92 & 87 & 89 & 89 & 88 \\
\hline$M$ & 78 & 78 & 80 & 79 & 76 & 79 & 83 & 80 & 82 & 80 \\
\hline
\end{tabular}

Note-Numerals indicate percent correct responses in 100-trial blocks.

dala ablations, similar to those used in the study by Murray and Mishkin (1985), but in monkeys trained in the same manner as those in Experiment 1.

\section{Method}

\section{Subjects}

The subjects were 4 cynomolgus monkeys (Macaca fascicularis). Two of them were subjects from Experiment $1-$ one (BL4) from Group BL and one (CM-4) from Group CM-each chosen for having achieved the best postoperative cross-modal score in its group. For clarity, in Experiment 2, we will refer to BL-4 and CM-4 as $A-3 / B L-4$ and $A-4 / C M-4$, respectively. The other 2 subjects were experimentally naive monkeys: 1 female and 1 male, weighing 3.5 and $4.5 \mathrm{~kg}$, respectively, at the beginning of the study. The monkeys were housed and fed in the manner described in Experiment 1.

\section{Apparatus and Materials}

The apparatus and materials were identical to those used in Experiment 1 .

\section{Training Procedures}

As described in Experiment 1, Subjects A-3/BL-4 and A-4/ CM-4 were trained preoperatively on visual and tactual DNMS to criterion and were then given pre- and postoperative testing on cross-modal DNMS. Their total training on the latter task, including the preoperative baseline, was 900 and 1,300 trials, respectively. The 2 new monkeys (A-1 and A-2) were trained using the same procedure as in Experiment 1, but then, to match the total amount of training given the other 2 monkeys, they were tested preoperatively on cross-modal DNMS for 1,000 trials, including the preoperative baseline.

\section{Surgery}

All 4 monkeys then received the same ablations, which were the only surgeries performed on A-1 and A-2 but constituted extensions of the excitotoxic lesions made previously in A-3/BL-4 and $A-4 / C M-4$. The monkeys were anesthetized with ketamine hydrochloride $(10 \mathrm{mg} / \mathrm{kg}$ ) followed by Nembutal, which was administered intravenously as required (approximately $20-30 \mathrm{mg} / \mathrm{kg}$ ). A solution of $5 \%$ dextrose and $0.45 \%$ sodium chloride was given as an intravenous drip, and heart rate, respiration rate, and temperature were monitored throughout surgery. A bone opening was made in the appropriate portion of the cranium; bilateral surgical ablations, carried out with the aid of an operating microscope, were performed aseptically in a single stage.

The amygdala plus subjacent cortex was removed via an orbital frontal approach. The frontal lobe was gently elevated slightly, and the tissue just medial to the anterior tip of the rhinal sulcus was entered with a small-gauge sucker. The amygdala was then removed by aspiration, the boundaries of the ablation consisting of the fundus of the rhinal sulcus ventrolaterally, the white matter of the temporal stem laterally, and the hippocampus caudally. The intended lesion thus included not only the amygdaloid complex but also the piriform cortex, periamygdaloid cortex, and, roughly, the rostral half of the entorhinal cortex (i.e., the cortical areas located rostral, medial, and ventral to the amygdala).

After the removals were completed, the dura mater was sutured, the scalp was closed in anatomical layers, and an antibiotic was given as a prophylactic measure.

\section{Postoperative Testing}

Postoperative training was initiated $2-4$ weeks after surgery. The monkeys received a total of 300 trials on the cross-modal version of DNMS, administered in the same manner as it had been preoperatively. The monkeys were then retrained to criterion first on visual DNMS and then on tactual DNMS. Unlike the monkeys in Experiment 1, however, these started retraining on the tactual task immediately after attaining criterion on visual DNMS, without the light-dimming phase between them. The criterion for mastering both the visual and the tactual tasks was the same as preoperatively (i.e., 90 correct responses in 100 consecutive trials). The monkeys that did not attain criterion on tactual DNMS within the 1,000-trial training limit were given training with double-sample presentation (as described in Experiment 1). After the monkeys had reached criterion on both intramodal versions of DNMS, they were retested on the cross-modal version for an additional 500 trials. The monkeys that required double-sample presentation to learn tactual DNMS also received this procedure for the subsequent retesting on cross-modal DNMS.

\section{Histology}

All 4 monkeys sustained complete damage to the amygdala bilaterally. In all cases, the lesion included the anterior portion of the entorhinal cortex, as intended. The location and extent of the damage is shown for two representative cases in Figure 2. As indicated in Table 4, there was inadvertent partial bilateral damage to the perirhinal cortex in two monkeys (A-1 and A-4/CM-4). The lesions also invaded the white matter adjacent to the amygdala either unilaterally (A-1 and A-3/BL-4) or bilaterally (A-2 and A-4/ 


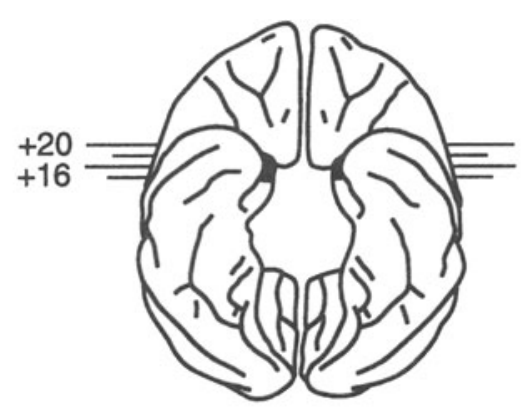

Case A-3/BL-4
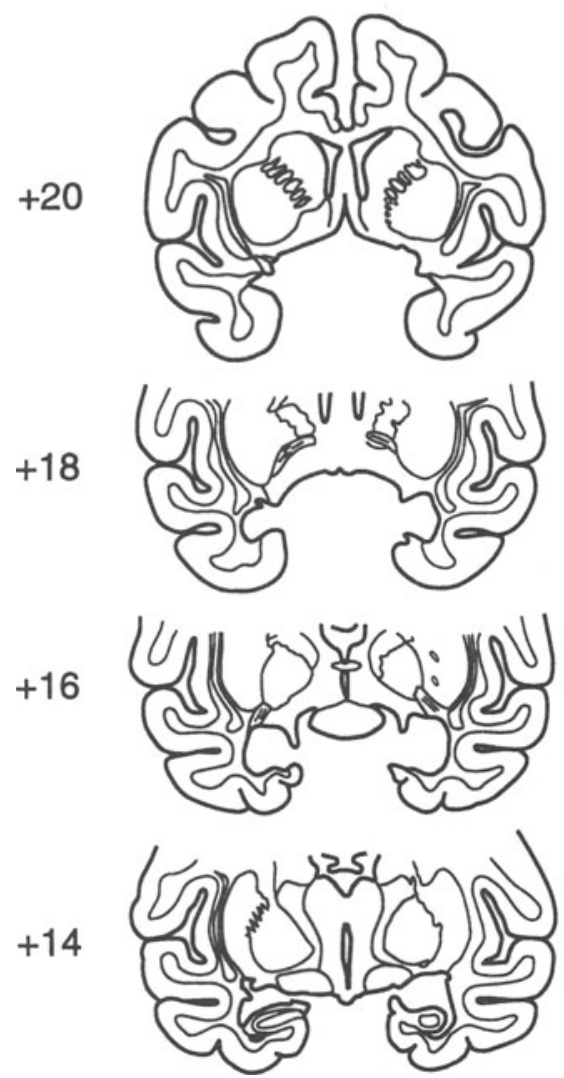

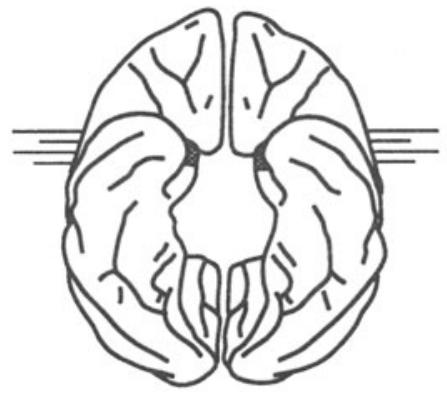

Intended Lesion
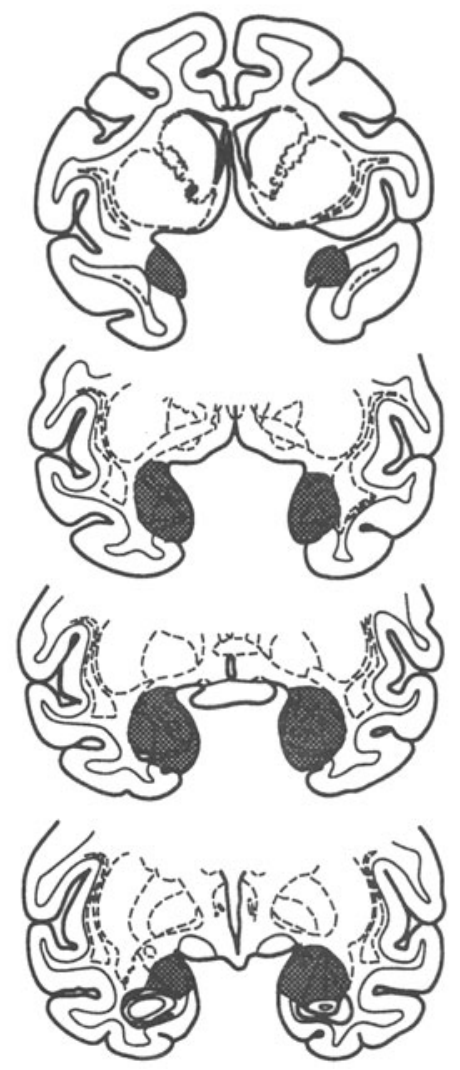

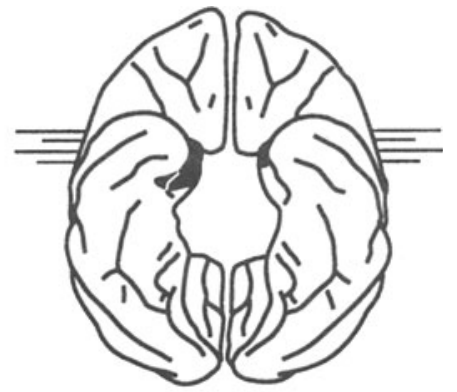

Case A-1
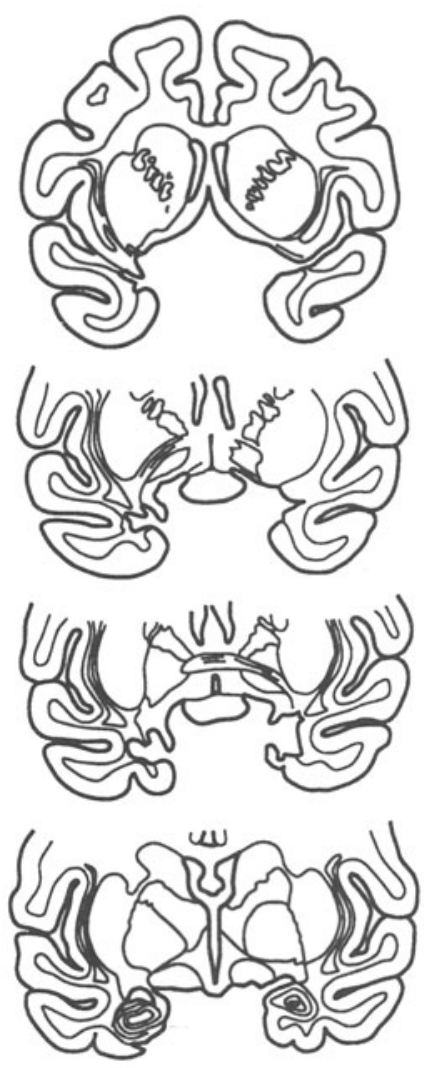

Figure 2. Surface reconstruction of the amygdala lesion on a standard ventral view of a macaque brain (top) and coronal sections through the lesion (below). The intended lesion (middle column) is indicated by the shaded regions on the ventral view and coronal sections of a normal brain. The lesions in two representative cases from Experiment 2 are shown on the ventral view (black) and coronal sections in the left and right columns. Numerals indicate the distance in millimeters from the interaural plane (0).

CM-4). In addition, all monkeys sustained damage to the tail of the caudate nucleus; this damage extended along the entire length of the tail bilaterally in A-1 and unilaterally in A-3/BL-4; the other 2 monkeys sustained partial bilateral damage, which extended, in A-2, $11 \mathrm{~mm}$ in anteroposterior extent on the left side and $4 \mathrm{~mm}$ on the right, and, in A-4/CM-4, $5 \mathrm{~mm}$ on each side. The damage to the tail of the caudate nucleus was associated with some cell loss and gliosis in the ventral putamen on the right in A-4/CM-4 and bilaterally in A-3/BL-4. Finally, 2 monkeys sustained slight damage to the hippocampus: $1(\mathrm{~A}-1)$ to the left hippocampus for a distance of $3 \mathrm{~mm}$ anteroposteriorly, and one $(\mathrm{A}-3 / \mathrm{BL}-4)$ mainly in the $\mathrm{CA} 3$ region and dentate gyrus, bilaterally for $3 \mathrm{~mm}$. The damage to the hippocampus in the latter case appeared to be a result of the ibotenic acid injections administered in Experiment 1.

\section{Results}

Preoperative Learning and Performance

The preoperative learning scores of the 2 new animals (A-1 and A-2) in both the visual and the tactual DNMS, as well as their performance in the cross-modal task, fell within the range of scores of the monkeys in Experiment 1 (Table 2). The 4 monkeys achieved an average of $83 \%$ correct responses on cross-modal DNMS.

\section{Postoperative Performance and Relearning}

Cross-modal DNMS. As shown in Table 5 and Figure 3 , the scores of all 4 monkeys fell significantly, rel- 
Table 4

Percent Damage to Entorhinal (ERh), Perirhinal (PRh), and Rhinal (Rh) Cortex

\begin{tabular}{lccc}
\hline Subject & ERh & PRh & Rh \\
\hline A-1 & 56 & 18 & 37 \\
A-2 & 30 & 1 & 16 \\
A-3/BL-4 & 33 & 4 & 19 \\
A-4/CM-4 & 36 & 38 & 37 \\
\hline
\end{tabular}

Note--Numerals indicate the mean percent damage sustained in the two hemispheres, expressed as a percent of the total volume. Rhinal cortex $(\mathrm{Rh})$ refers to the entorhinal and perirhinal cortex combined, each of which comprises close to $50 \%$ of the rhinal cortical volume.

ative to their own preoperative levels [mean score of $57 \%$ vs. $83 \% ; t(3)=5.57, p<.01]$.

Relearning of visual and tactual DNMS. All animals relearned visual DNMS to criterion, 1 (A-2) requiring more trials and 3 (A-1, A-3/BL-4, and A-4/CM-4) requiring fewer trials than they had required to learn the task preoperatively (Table 6). On tactual DNMS, only 1 animal (A-3/BL-4) met the criterion within the training limit of 1,000 trials. Two of the animals (A-1 and A-2) that required additional training with double-sample presentation improved immediately to the level of $88 \%$ and $87 \%$ correct, at which point retraining was stopped. The 4th animal (A-4/CM-4) became disruptive during testing, and retraining was discontinued after 840 trials $(60 \%$ correct in the last 100 trials).

Cross-modal DNMS retesting. The 3 remaining monkeys scored an average of $4.5 \%$ higher than on the initial test immediately after surgery; the improvement was significant [paired $t$ test, $t(2)=6.50, p<.03$ ]. As shown in Figure 3, however, average performance on the retest was still $19 \%$ lower than the preoperative performance $(81 \%)$, the difference still constituting a significant impairment, as predicted [paired $t$ test $t(2)=3.34, p<.05$, one-tailed].

\section{Comment}

The results of Experiment 2 confirm the results of Murray and Mishkin (1985) in showing that bilateral aspiration lesions of the amygdala plus subjacent cortex significantly disrupt performance on cross-modal DNMS. Thus, the possibility raised earlier, that the lack of effect of the subtotal amygdalar lesions in Experiment 1 was due to differences in training procedures in the two studies, can be ruled out.

\section{DISCUSSION}

Before the effects of the amygdalar lesions are considered, a comment is in order regarding preoperative levels of performance on the cross-modal task. In the earlier cross-modal study by Murray and Mishkin (1985), no normal monkeys were tested, but a group of 3 hippocampectomized monkeys achieved an average of $90 \%$ correct responses over the 500 trials of cross-modal DNMS, immediately upon transfer from the tactile DNMS. It was assumed at the time that this represented normal performance. We were therefore surprised that the intact monkeys in the present experiments had such difficulty in mastering the cross-modal task and also performed relatively poorly ( $80 \%$ correct). This disparity in the scores raises the possibility that hippocampal removals actually facilitate performance on the cross-modal task. It is of interest in this connection that hippocampectomized rats perform better on olfactory paired associates than do controls (Eichenbaum \& Bunsey, 1995), another type of stimulusstimulus associative memory.

As indicated above, the level of performance achieved by the normal animals on cross-modal DNMS was $80 \%$ correct. This level was not affected by the subtotal lesions of the amygdala (Experiment 1). By contrast, aspiration lesions of the amygdala that included the subjacent cortex consistently impaired performance (Experiment 2; see also Murray \& Mishkin, 1985). In these latter cases, intramodal abilities were not entirely spared. In Experiment 2, for example, 3 of the 4 amygdalectomized monkeys took more trials to relearn tactual DNMS than they had taken in initial learning, and 1 of these was similarly retarded in relearning visual DNMS. Nevertheless, there was a differential effect on cross-modal DNMS, in that this impairment followed reattainment of criterion in the intramodal tasks.

Table 5

Preoperative and Postoperative Learning and Performance

\begin{tabular}{|c|c|c|c|c|c|c|c|c|c|c|c|c|c|c|}
\hline \multirow[b]{3}{*}{ Subject } & \multicolumn{10}{|c|}{ Preoperative } & \multirow{2}{*}{\multicolumn{4}{|c|}{$\begin{array}{l}\text { Postoperative } \\
\text { Cross-Modal } \\
\text { Performance }\end{array}$}} \\
\hline & \multicolumn{2}{|c|}{$\begin{array}{l}\text { Visual } \\
\text { DNMS }\end{array}$} & \multicolumn{2}{|c|}{$\begin{array}{l}\text { Tactual } \\
\text { DNMS }\end{array}$} & \multicolumn{2}{|c|}{$\begin{array}{c}\text { Cross-Modal } \\
\text { DNMS }\end{array}$} & \multicolumn{4}{|c|}{$\begin{array}{l}\text { Cross-Modal } \\
\text { Performance }\end{array}$} & & & & \\
\hline & Trials & Errors & Trials & Errors & Trials & Errors & 100 & 200 & 300 & $M$ & 100 & 200 & 300 & $M$ \\
\hline A-1 & 580 & 157 & 420 & 89 & 700 & 211 & 80 & 74 & 76 & 77 & 68 & 62 & 61 & 64 \\
\hline$A-2$ & 260 & 60 & 560 & 89 & 700 & 160 & 80 & 80 & 75 & 79 & 44 & 51 & 53 & 49 \\
\hline A-3/BL-4 & 180 & 37 & 360 & 51 & 600 & 76 & 82 & 92 & 89 & 88 & 59 & 60 & 61 & 60 \\
\hline A-4/CM-4 & 220 & 58 & 400 & 80 & 1,000 & 163 & 87 & 89 & 89 & 88 & 53 & 50 & 56 & 53 \\
\hline$M$ & 310 & 78 & 435 & 77 & 750 & 153 & 82 & 84 & 82 & 83 & 56 & 56 & 58 & 57 \\
\hline
\end{tabular}

Note - The total number of trials and errors scored in the three stages of training, together with the pre- and postoperative performance scores on cross-modal DNMS. Subjects A-3 and A-4 participated in Experiment 1 . As a result, the learning scores for these 2 subjects are the same as those provided in Table 2 , with the addition of the 300 -trial preoperative baseline and the first 200 trials for postoperative performance. In addition, their performance scores prior to the second operation are the same as those listed under the last 300 trials of Postoperative Performance in Table 3 . For all monkeys, the number of trials listed under cross-modal DNMS excludes the 300-trial preoperative baseline. 


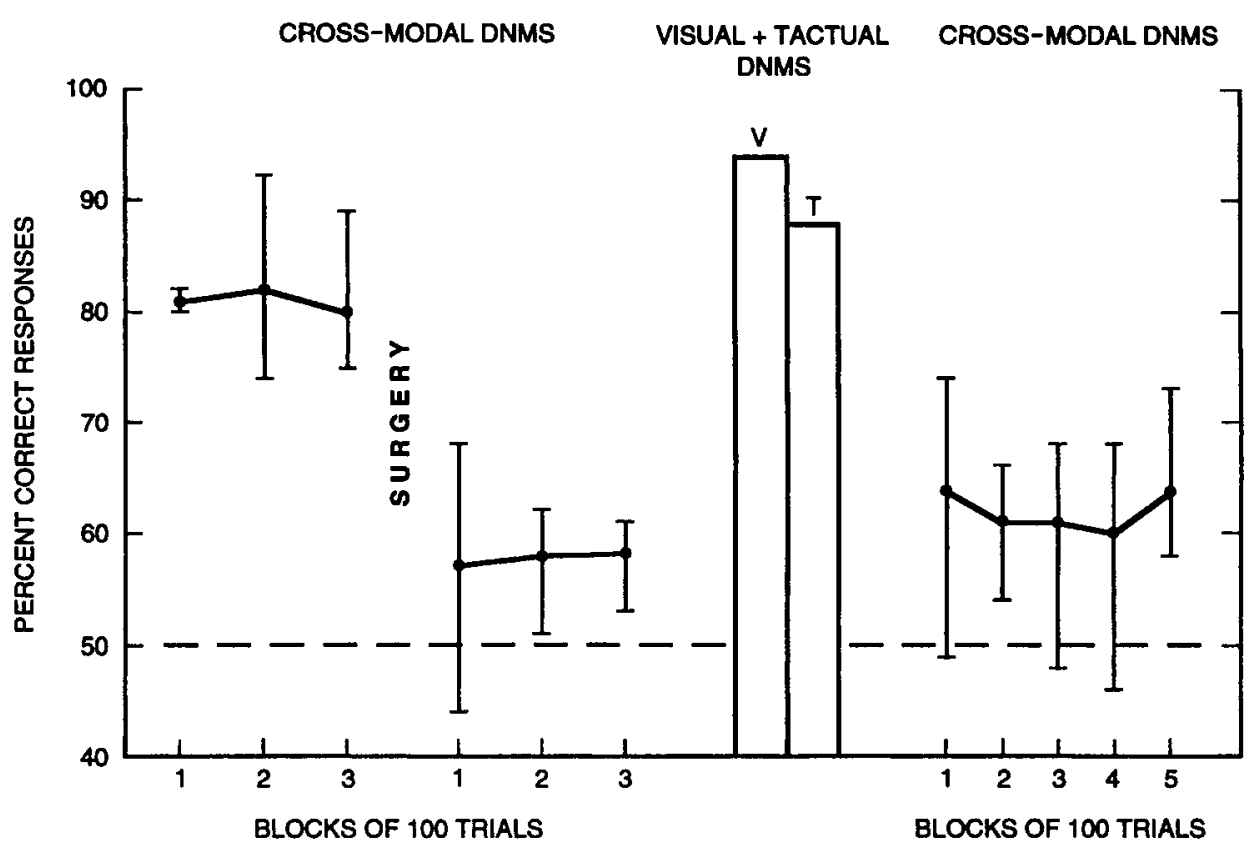

Figure 3. Average scores on cross-modal delayed nonmatching-to-sample (DNMS) or on visual (V) and tactual ( $T$ ) versions of the same task obtained by monkeys in Experiment 2. The first three blocks of trials indicate the average performance during the 300 trials of the preoperative baseline. The second three blocks indicate the average performance during 300 trials postoperatively. The last five blocks show performance on the cross-modal retest after the relearning of the visual and the tactual DNMS. Vertical lines indicate the range of scores on cross-modal DNMS.

The lack of effect of damaging either the basolateral or the centromedial subdivisions of the amygdala in Experiment 1 may have been due to the incompleteness of the lesions. However, there was no relationship between the extent of damage to either subdivision and behavioral outcome. Even the monkey with $70 \%$ damage to the lateral basal nucleus, the major source of direct basolateral projections to the neocortex, showed no impairment. Examination of the histological material reported by Streicher and Ettlinger (1987) suggests that their "small" amygdala lesions involved more of the basolateral group than ours did, yet still had no effect. Their "more extensive" lesions (e.g., AMYG-2) involved the entorhinal and peri- rhinal cortex, the same regions compromised in the monkeys in Experiment 2 in the present study.

Because aspiration lesions of amygdala include several components - namely, the amygdala, anterior entorhinal cortex, anterior perirhinal cortex, periamygdaloid cortex, and piriform cortex-it is still not possible to identify the critical neural substrate for cross-modal associations. The severe deficit could have been produced by (1) complete amygdala lesions, (2) damage to structures nearby the amygdala, such as the entorhinal cortex, perirhinal cortex, or piriform cortex, or (3) damage to the amygdala in combination with some or all of the surrounding structures.

Table 6

Postoperative Relearning and Performance

\begin{tabular}{|c|c|c|c|c|c|c|c|c|c|c|c|c|}
\hline \multirow[b]{2}{*}{ Subject } & \multicolumn{3}{|c|}{ Visual DNMS } & \multicolumn{3}{|c|}{ Tactual DNMS } & \multicolumn{6}{|c|}{ Cross-Modal DNMS Performance } \\
\hline & Trials & Errors & $\%$ & Trials & Errors & $\overline{\%}$ & 100 & 200 & 300 & 400 & 500 & $\bar{M}$ \\
\hline$\overline{A-1}$ & 540 & 112 & 93 & $1,020^{*}$ & 367 & 88 & 74 & 66 & 67 & 67 & 73 & 69 \\
\hline A-2 & 680 & 160 & 89 & $1,020^{*}$ & 19 & 87 & 49 & 54 & 48 & 46 & 61 & 52 \\
\hline A- $3 /$ BL- 4 & 0 & 0 & 100 & 280 & 69 & 90 & 68 & 64 & 68 & 68 & 58 & 65 \\
\hline $\mathrm{A}-4 / \mathrm{CM}-4 \dagger$ & 80 & 13 & 90 & & & & & & & & & \\
\hline$M$ & 325 & 71 & 93 & 773 & 152 & 88 & 64 & 61 & 61 & 60 & 64 & 62 \\
\hline
\end{tabular}

Note-Numerals indicate number of trials and errors to criterion and score (\%) in last 100 trials on visual and tactual DNMS, and percent correct responses in blocks of 100 trials on crossmodal DNMS. *Failed to attain criterion within 1,000 trials; double-sample presentation was then given, and performance rose to criterion level after 20 trials. †Subject A-4/CM-4 failed to attain criterion within 840 trials on tactual DNMS and was excluded from further analyses. 
Although several of these structures could contribute to cross-modal associative memory, there is mounting evidence pointing to a special role for the entorhinal and perirhinal, or rhinal, cortex (Murray, 1996). The rhinal cortex, but not the amygdala or hippocampus, is important for both stimulus identification (Eacott, Gaffan, \& Murray, 1994) and stimulus recognition (Horel, PytkoJoiner, Voytko, \& Salsbury, 1987; Meunier, Bachevalier, Mishkin, \& Murray, 1993; Mishkin \& Murray, 1994; Murray 1996; Murray, E. A. Gaffan, \& Flint, 1996; ZolaMorgan, Squire, Amaral, \& Suzuki, 1989) - findings that are consistent with the difficulty displayed by the monkeys in Experiment 2 in relearning the intramodal versions of DNMS. Furthermore, the one animal (BL-3) to show a sizable drop in scores postoperatively in Experiment 1 (see Table 2) was the only one with bilateral damage to the entorhinal cortex. Although this damage was slight, the possibility that it contributed to this animal's drop in performance cannot be ruled out. Finally, the rhinal cortex, but not the amygdala or the hippocampus, is critical for the learning of intramodal (visual) stimulusstimulus associations (Murray, D. Gaffan, \& Mishkin, 1993). These several findings, taken together with the negative results of Experiment 1 and the positive results of Experiment 2, raise the possibility that the rhinal cortex, rather than the amygdala, is the critical neural substrate for cross-modal recognition.

\section{REFERENCES}

AgGleton, J. P. (1985). X-ray localization of limbic structures in the cynomolgus monkey (Macaca fascicularis). Journal of Neuroscience Methods, 14, 101-108.

Aggleton, J. P., Burton, M. J., \& Passingham, R. E. (1980). Cortical and subcortical afferents to the amygdala of the rhesus monkey (Macaca mulatta). Brain Research, 190, 347-368.

Aggleton, J. P., \& Mishrin, M. (1983). Memory impairments following restricted medial thalamic lesions in monkeys. Experimental Brain Research, 52, 199-209.

AgGleton, J. P., \& Mishin, M. (1984). Projections of the amygdala to the thalamus in the cynomolgus monkey. Journal of Comparative Neurology, 222, 56-68.

Aggleton, J. P., \& Passingham, R. E. (1981). Stereotaxic surgery under $\mathrm{X}$-ray guidance in the rhesus monkey, with special reference to the amygdala. Experimental Brain Research, 44, 271-276.

Amaral, D. G., Insausti, R., \& Cowan, W. M. (1983). Evidence for a direct projection from the superior temporal gyrus to the entorhinal cortex in the monkey. Brain Research, 275, 263-277.

Amaral, D. G., \& Price, J. L. (1984). Amygdalo-cortical projections in the monkey (Macaca fascicularis). Journal of Comparative Neurology, 230, 465-496.

Barbas, H., \& De Olmos, J. (1990). Projections from the amygdala to basoventral and mediodorsal prefrontal regions in the rhesus monkey. Journal of Comparative Neurology, 300, 549-571.

Bolster, R. B. (1978). Cross-modal matching in the monkey (Macaca fascicularis). Neuropsychologia, 16, 407-416.

CoWey, A., \& Weiskrantz, L. (1975). Demonstration of cross-modal matching in rhesus monkeys (Macaca mulatta). Neuropsychologia, 13, 117-120.

EacotT, M. J., Gaffan, D., \& Murray, E. A. (1994). Preserved recognition memory for small sets, and impaired stimulus identification for large sets, following rhinal cortex ablations in monkeys. European Journal of Neuroscience, 6, 1466-1478.

EICHENBAUM, H., \& BunSEY, M. (1995). On the binding associations in memory: Clues from studies on the role of the hippocampal region in paired-associate learning. Current Directions in Psychological Science, 4, 19-23.

ELLIOT, R. C. (1977). Cross-modal recognition in three primates. Neuropsychologia, 15, 183-186.

Friedman, D. P., Murray, E. A., O'Neill, J. B., \& Mishin, M. (1986). Cortical connections of the somatosensory fields of the lateral sulcus of macaques: Evidence for a corticolimbic pathway for touch. Journal of Comparative Neurology, 252, 323-347.

GafFan, D., \& Murray, E. A. (1990). Amygdalar interaction with the mediodorsal nucleus of the thalamus and the ventromedial prefrontal cortex in stimulus-reward associative learning in the monkey. Journal of Neuroscience, 10, 3479-3493.

Herzog, A. G., \& VAN Hoesen, G. W. (1976). Temporal neocortical afferent connections to the amygdala in the rhesus monkey. Brain Research, 115, 57-69.

Horel, J. A., Pytko-Joiner, D. E., Voytko, M. L., \& Salsbury, K. (1987). The performance of visual tasks while segments of the inferior temporal cortex are suppressed by cold. Behavioral Brain Research, 23, 29-42.

IWAI, E., \& YUKIE, M. (1987). Amygdalofugal and amygdalopetal connections with modality-specific visual cortical areas in macaques (Macaca fuscata, M. mulatta, and M. fascicularis). Journal of Comparative Neurology, 261, 362-368.

JARVIS, M. J., \& ETTLINGER, G. (1977). Cross-modal recognition in chimpanzees and monkeys. Neuropsychologia, 15, 499-506.

Meunier, M., Bachevalier, J., Mishkin, M., \& Murray, E. A. (1993). Effects of visual recognition on combined and separate ablations of the entorhinal and perirhinal cortex in rhesus monkeys. Journal of Neuroscience, 13, 5418-5432.

Mishkin, M., \& MurRay, E. A. (1994). Stimulus recognition. Current Opinion in Neurobiology, 4, 200-206.

Morecraft, R. J., Geula, C., \& Mesulam, M.-M. (1992). Cytoarchitecture and neural afferents of orbitofrontal cortex in the brain of the monkey. Journal of Comparative Neurology, 323, 341-358.

Mufson, E. J., Mesulam, M.-M., \& Pandya, D. N. (1981). Insular interconnections with the amygdala in the rhesus monkey. Neuroscience, 6, 1231-1248.

MurRay, E. A. (1996). What have the ablation studies told us about the neural substrate of stimulus memory? Seminars in the Neurosciences, 8, 13-22.

Murray, E. A., Gaffan, D., \& Mishinin, M. (1993). Neural substrates of visual stimulus-stimulus association in rhesus monkeys. Journal of Neuroscience, 13, 4549-4561.

Murray, E. A., Gaffan, E. A., \& Flint, R. W., Jr. (1996). Anterior rhinal cortex and amygdala: Dissociation of their contributions to memory and food preference in rhesus monkeys. Behavioral Neuroscience, 110, 30-42.

MurRay, E. A., \& Mishrin, M. (1985, May 3). Amygdalectomy impairs crossmodal associations in monkeys. Science, 228, 604-606.

Petrides, M., \& Iversen, S. D. (1976, June 4). Cross-modal matching and the primate frontal cortex. Science, 192, 1023-1024.

Russchen, F. T., Amaral, D. G., \& Price, J. L. (1987). The afferent input to the magnocellular division of the mediodorsal thalamic nucleus in the monkey (Macaca fascicularis). Journal of Comparative Neurology, 256, 175-210.

Streicher, M., \& EtTlinger, G. (1987). Cross-modal recognition of familiar and unfamiliar objects by the monkey: The effects of ablation of polysensory neocortex of the amygdaloid complex. Behavioural Brain Research, 23, 95-107.

Tigges, J., Tigges, M., Cross, N. A., McBride, R. L., Letbetter, W. D., \& ANSCHEL, S. (1982). Subcortical structures projecting to visual cortical areas in the monkey. Journal of Comparative Neurology, 209, $29-40$.

TURner, B. H., Mishkin, M., \& KNAPP, M. (1980). Organization of amygdalopetal projections from modality-specific cortical association areas in the monkey. Journal of Comparative Neurology, 191, 515-543.

VAN HOESEN, G. W. (1981). The differential distribution, diversity and sprouting of cortical projections to the amygdala in the rhesus mon- 
key. In Y. Ben-Ari (Ed.), The amygdaloid complex (pp. 77-90). Amsterdam: Elsevier/North-Holland.

Weiskrantz, L., \& Cowey, A. (1975). Cross-modal matching in the rhesus monkey using a single pair of stimuli. Neuropsychologia, 13, 257-261.

Zola-Morgan, S., \& SQuire, L. R. (1985). Amnesia in monkeys after lesions of the mediodorsal nucleus of the thalamus. Annals of Neurology, 17, 558-564.
Zola-Morgan, S., Squire, L. R., Amaral, D. G., \& Suzuki, W. A. (1989). Lesions of perirhinal and parahippocampal cortex that spare the amygdala and hippocampal formation produce severe memory impairment. Journal of Neuroscience, 9, 4355-4370.

(Manuscript received April 25, 1996; revision accepted for publication July 14, 1996.) 